\title{
NONVANISHING MEROMORPHIC UNIVALENT FUNCTIONS
}

\author{
YUSUF ABU-MUHANNA AND GLENN SCHOBER
}

(Communicated by Irwin Kra)

\begin{abstract}
This note studies the best constants $s$ such that the function $k(z)=z+2+1 / z$ solves the linear coefficient problems $\max \operatorname{Re}\left\{s b_{0}+b_{n}\right\}$ and $\max \operatorname{Re}\left\{s b_{0}-b_{n}\right\}$ over nonvanishing functions in the class $\Sigma$.
\end{abstract}

Let $\Sigma$ be the class of functions $f(z)=z+\sum_{n=0}^{\infty} b_{n} z^{-n}$ that are analytic and univalent in $\{z:|z|>1\}$. The coefficient problem for this class appears to be difficult. A sharp bound for $\operatorname{Re} b_{n}$ is known only for $1 \leq n \leq 3$, although there are some conjectures $[10,12]$ for larger $n$. One obstacle seems to be that the extremal functions change with $n$. Another is their nonelementary nature. To avoid these obstacles, one may consider some linear problems for which the elementary functions $k\left(z ; b_{0}\right)=z+b_{0}+1 / z$ are extremal.

For example, let us consider the functionals $\operatorname{Re}\left\{t b_{1} \pm b_{n}\right\}$ for fixed $n \geq 2$ and $t>0$. It was shown in [5] that the maximum of these functionals is attained by the functions $k\left(\cdot ; b_{0}\right)$ for all $t$ sufficiently large. That is, $\operatorname{Re}\left\{t b_{1} \pm b_{n}\right\} \leq t$ are valid inequalities for all $t$ sufficiently large. How large $t$ must be depends on $n$. Therefore it makes sense to define

$$
\mathscr{A}_{n}=\inf \left\{t: \operatorname{Re}\left(t b_{1}+b_{n}\right) \leq t\right\}
$$

and

$$
\mathscr{B}_{n}=\inf \left\{t: \operatorname{Re}\left(t b_{1}-b_{n}\right) \leq t\right\}
$$

for $n \geq 2$. The infimum is over all $t$ such that the given inequality is valid for all functions in $\Sigma$. Each infimum is a finite positive number and is actually a minimum. Since $-f(-z)$ is in $\Sigma$ whenever $f$ is, it is clear that $\mathscr{A}_{n}=\mathscr{B}_{n}$ for even $n$. The following is a summary of what is known about the numbers $\mathscr{A}_{n}$ and $\mathscr{B}_{n}$. Proofs of various parts are contained in the references cited, and a survey appears in $[11]$.

THEOREM $1 . \mathscr{A}_{2}=\mathscr{B}_{2}=2 \quad[\mathbf{1}, \mathbf{3}]$, $\mathscr{A}_{3}=\left(e^{4}+3\right) /\left(e^{4}-1\right) \quad[8,6]$,

$\mathscr{B}_{3}=3 \quad[\mathbf{1}, \mathbf{3}]$,

$5 / 4 \leq \mathscr{A}_{5} \leq(27+8 \sqrt{3}) / 12 \quad[7,6]$,

$1.6311<\mathscr{A}_{7} \leq 5.5 \quad[7,6]$,

$2.0391<\mathscr{A}_{9}<8 \quad[\mathbf{7}, \mathbf{6}]$,

$2.4575<\mathscr{A}_{11}<10 \quad[7,6]$,

Received by the editors June 30, 1987 and, in revised form, September 23, 1987.

1980 Mathematics Subject Classification (1985 Revision). Primary 30C50.

The work of the second author was supported in part by a grant from the National Science Foundation.

(C) 1988 American Mathematical Society $0002-9939 / 88 \$ 1.00+\$ .25$ per page 


$$
\begin{aligned}
\frac{n}{2}\left[1+(-1)^{n}\right] & +\frac{n}{3 \pi}\left[1-(-1)^{n}\right] \\
& \leq \mathscr{A}_{n} \leq \frac{1}{4}\left(4 n^{4}+50 n^{3}+149 n^{2}+277 n+6\right) 3^{n-5} \quad[\mathbf{5}, \mathbf{6}], \\
n \leq \mathscr{B}_{n} & \leq \frac{1}{4}\left(4 n^{4}+50 n^{3}+149 n^{2}+277 n+6\right) 3^{n-5} \quad[\mathbf{5}, \mathbf{6}] .
\end{aligned}
$$

In addition, it is known [12] that the inequality $\operatorname{Re}\left\{t b_{1}+\left|b_{n}\right|\right\} \leq t$ is valid for all functions $f \in \Sigma$ with $\operatorname{Re}\left\{b_{1}\right\} \leq\left(n t^{2}-1\right) /\left(n t^{2}+1\right)$.

In this note we shall consider the family $\Sigma^{\prime}$ of functions in $\Sigma$ which omit the value zero. A prominent function in this family is $k(z) \equiv k(z ; 2)=z+2+1 / z$. Since $\operatorname{Re}\left\{b_{0}\right\} \leq 2$ for all functions in $\Sigma^{\prime}$, it is conceivable that the maximum of the functionals $\operatorname{Re}\left\{s b_{0} \pm b_{n}\right\}$ over $\Sigma^{\prime}$ would be attained by the function $k$ for all $s$ sufficiently large. For this reason let us define

$$
\mathscr{A}_{n}^{\prime}=\inf \left\{s: \operatorname{Re}\left(s b_{0}+b_{n}\right) \leq 2 s\right\}
$$

and

$$
\mathscr{B}_{n}^{\prime}=\inf \left\{s: \operatorname{Re}\left(s b_{0}-b_{n}\right) \leq 2 s\right\}
$$

for $n \geq 2$, where the infimum is over all $s$ such that the given inequality is valid for all functions in $\Sigma^{\prime}$. At first glance, it is not obvious that $\mathscr{A}_{n}^{\prime}$ or $\mathscr{B}_{n}^{\prime}$ is finite.

It will be useful to have estimates for our functionals when $n=1$.

LEMMA 2. The following are sharp inequalities for functions in $\Sigma^{\prime}$.

(a) $\operatorname{Re}\left\{s b_{0}+b_{1}\right\} \leq 2|s|+1$, if $-\infty<s<\infty$,

(b) $\operatorname{Re}\left\{s b_{0}-b_{1}\right\} \leq 1+\frac{3}{8} s^{2}+\frac{1}{4} s^{2}(\log 4 /|s|)$ if $-4 \leq s \leq 4$,

(c) $\operatorname{Re}\left\{s b_{0}-b_{1}\right\} \leq 2|s|-1$ if $s<-4$ or $s>4$.

PROOF. Part (a) follows from the elementary inequalities $\left|b_{0}\right| \leq 2$ and $\left|b_{1}\right| \leq 1$. Part (b) is not so elementary and is due to J. A. Jenkins [2, Corollary 2]. Part (c) follows from (b) with $|s|=4$ and the relations

$$
\begin{aligned}
\operatorname{Re}\left\{s b_{0}-b_{1}\right\} & =\operatorname{Re}\left\{4 \frac{s}{|s|} b_{0}-b_{1}\right\}+\left[1-\frac{4}{|s|}\right] s \operatorname{Re}\left\{b_{0}\right\} \\
& \leq 7+\left[1-\frac{4}{|s|}\right] 2|s|=2|s|-1
\end{aligned}
$$

Parts (a) and (c) are sharp either for the function $k$ or for $-k(-z)$. Part (b) is sharp (see [2]) for certain functions in $\Sigma^{\prime}$ that map onto the complement of slits on the critical trajectories of the quadratic differentials $(|s| / w-1) d w^{2}$.

If $\mathscr{A}_{1}^{\prime}$ and $\mathscr{B}_{1}^{\prime}$ are defined in the same spirit relative to the function $k$, that is, $\mathscr{A}_{1}^{\prime}=\inf \left\{s: \operatorname{Re}\left(s b_{0}+b_{1}\right) \leq 2 s+1\right\} \quad$ and $\mathscr{B}_{1}^{\prime}=\inf \left\{s: \operatorname{Re}\left(s b_{0}-b_{1}\right) \leq 2 s-1\right\}$, then on the basis of Lemma 2 we may identify $\mathscr{A}_{1}^{\prime}=0$ and $\mathscr{B}_{1}^{\prime}=4$.

One consequence of the following theorem is that the constants $\mathscr{A}_{n}^{\prime}$ and $\mathscr{B}_{n}^{\prime}$ are finite.

THEOREM 3. For $n \geq 2$, we have

$$
\max _{\theta} \frac{-2 \sin (2 n \theta)}{\tan \theta} \leq \mathscr{A}_{n}^{\prime} \leq 4 \mathscr{A}_{n}
$$

and $4 n \leq \mathscr{B}_{n}^{\prime} \leq 4 \mathscr{B}_{n}$. 
ProOF. Let $\sigma= \pm 1$, and let $s$ be a value such that $\operatorname{Re}\left\{s b_{0}+\sigma b_{n}\right\} \leq 2 s$ is valid for the class $\Sigma^{\prime}$. Equality occurs for the function $k$. Thus if we apply boundary variations of the form $k^{*}=k+\varepsilon /(k-w)+\varepsilon / w+o(\varepsilon)$, which operate within the family $\Sigma^{\prime}$, it follows that $k$ must satisfy the corresponding Schiffer differential equation (cf. [9, Chapter 10])

$$
L\left[\frac{k}{k-w}\right] \frac{d w^{2}}{w} \geq 0
$$

where the functional $L$ picks out the combination of coefficients $s b_{0}+\sigma b_{n}$ from the function $k(z) /(k(z)-w)$ and $w$ is omitted by $k$. Since the function $k$ omits the real interval $[0,4]$, both $w$ and the differential $d w^{2}$ are positive for $0<w<4$. Therefore, if we parametrize $w=4 \cos ^{2} \theta, 0<\theta<\pi / 2$, then the differential equation implies

$$
L\left[\frac{k}{k-w}\right]=L\left[1+\sum_{j=1}^{\infty} \frac{2 \sin (2 j \theta)}{\tan \theta} z^{-j}\right]=s+2 \sigma \frac{\sin (2 n \theta)}{\tan \theta} \geq 0
$$

For $\sigma=-1, s=\mathscr{B}_{n}^{\prime}$, and $\theta \rightarrow 0$ we conclude that $\mathscr{B}_{n}^{\prime} \geq 4 n$. Similarly, for $\sigma=1$ and $s=\mathscr{A}_{n}^{\prime}$ we conclude that

$$
\mathscr{A}_{n}^{\prime} \geq \max _{\theta} \frac{-2 \sin (2 n \theta)}{\tan \theta}
$$

In order to derive the first upper bound we use the inequality $\operatorname{Re}\left\{\mathscr{A}_{n} b_{1}+b_{n}\right\} \leq$ $\mathscr{A}_{n}$, which is true by the definition of $\mathscr{A}_{n}$ and the fact that the infimum is a minimum, and the inequality $\operatorname{Re}\left\{4 b_{0}-b_{1}\right\} \leq 7$ from Lemma 2, part (b). Then the relations

$$
\operatorname{Re}\left\{s b_{0}+b_{n}\right\}=\frac{s}{4} \operatorname{Re}\left\{4 b_{0}-b_{1}\right\}+\operatorname{Re}\left\{\frac{s}{4} b_{1}+b_{n}\right\} \leq \frac{7 s}{4}+\frac{s}{4}=2 s
$$

are valid with $s=4 \mathscr{A}_{n} ;$ that is, $\mathscr{A}_{n}^{\prime} \leq 4 \mathscr{A}_{n}$. The proof that $\mathscr{B}_{n}^{\prime} \leq 4 \mathscr{B}_{n}$ is obtained by replacing $b_{n}$ by $-b_{n}$ and $\mathscr{A}_{n}$ by $\mathscr{\mathscr { B }}_{n}$.

By combining Theorems 1 and 3 we are able to identify or estimate some of the numbers $\mathscr{A}_{n}^{\prime}$ and $\mathscr{B}_{n}^{\prime}$.

\section{THEOREM 4.}

$$
\begin{gathered}
\mathscr{B}_{2}^{\prime}=8, \\
\mathscr{B}_{3}^{\prime}=12, \\
1 \leq \mathscr{A}_{2}^{\prime} \leq 8, \\
\frac{20+14 \sqrt{7}}{27} \leq \mathscr{A}_{3}^{\prime} \leq 4\left(e^{4}+3\right) /\left(e^{4}-1\right),
\end{gathered}
$$




$$
\begin{array}{rl} 
& 3.1068<\mathscr{A}_{4}^{\prime}, \\
& 4.0504<\mathscr{A}_{5}^{\prime} \leq(27+8 \sqrt{3}) / 3 \\
& 4.9688<\mathscr{A}_{6}^{\prime}, \\
& 5.8729<\mathscr{A}_{7}^{\prime} \leq 22, \\
& 6.7682<\mathscr{A}_{8}^{\prime}, \\
& 7.6576<\mathscr{A}_{9}^{\prime}<32, \\
& 8.5428<\mathscr{A}_{10}^{\prime}, \\
9 & .4251<\mathscr{A}_{11}^{\prime}<40, \\
2 \cot \left[\frac{3 \pi}{4 n}\right] \leq \max _{\theta} \frac{-2 \sin (2 n \theta)}{\tan \theta} \leq \mathscr{A}_{n}^{\prime} \leq\left(4 n^{4}+50 n^{3}+149 n^{2}+277 n+6\right) 3^{n-5}, \\
4 n \leq \mathscr{B}_{n}^{\prime} \leq\left(4 n^{4}+50 n^{3}+149 n^{2}+277 n+6\right) 3^{n-5} .
\end{array}
$$

PROOF. The estimates from above are all equal to four times the corresponding estimates from Theorem 1 , as asserted by Theorem 3 . For $\mathscr{B}_{2}^{\prime}$ and $\mathscr{B}_{3}^{\prime}$ these estimates coincide with the estimates from below in Theorem 3 .

For $n=2$, the expression $-2 \sin (2 n \theta) / \tan \theta$ from Theorem 3 reduces to $8 \cos ^{2} \theta-$ $16 \cos ^{4} \theta$. Its maximum occurs when $\cos ^{2} \theta=\frac{1}{4}$ and is the lower bound given for $\mathscr{A}_{2}^{\prime}$. For $n=3$, the expression $(-2 \sin (2 n \theta)) / \tan \theta$ becomes $-12 \cos ^{2} \theta+64 \cos ^{4} \theta-$ $64 \cos ^{6} \theta$. Its maximum occurs when $\cos ^{2} \theta=(4+\sqrt{7}) / 12$ and leads to the lower bound given for $\mathscr{A}_{3}^{\prime}$. For larger $n$ the maximum of $(-2 \sin (2 n \theta)) / \tan \theta$ can be approximated numerically, and these values are listed for $4 \leq n \leq 11$. In general, the choice $\theta=3 \pi / 4 n$ gives the lower bound $2 \cot [3 \pi / 4 n]$, which is of order $8 n / 3 \pi$ as $n \rightarrow \infty$, for $\mathscr{A}_{n}^{\prime}$.

The first constant not determined in Theorem 4 is $\mathscr{A}_{2}^{\prime}$. Although its value is still unknown, we can improve the upper bound considerably.

THEOREM 5. $1 \leq \mathscr{A}_{2}^{\prime} \leq 3$.

ProOF. We shall use the Grunsky inequalities (cf. [9, p. 118])

$$
\left|\sum_{\mu, \nu=1}^{N} \gamma_{\mu \nu} \lambda_{\mu} \lambda_{\nu}\right| \leq \sum_{\nu=1}^{N} \frac{1}{\nu}\left|\lambda_{\nu}\right|^{2} \quad\left(\lambda_{1}, \ldots, \lambda_{N} \in \mathbf{C}\right)
$$

for the coefficients $\gamma_{\mu \nu}$ of functions $g \in \Sigma$ generated by

$$
\log \frac{g(z)-g(\varsigma)}{z-\varsigma}=\sum_{\mu, \nu=1}^{\infty} \gamma_{\mu \nu} z^{-\mu} \varsigma^{-\nu}
$$

In particular, we shall use the inequality $\left|\gamma_{33}\right| \leq \frac{1}{3}$. If $f \in \Sigma^{\prime}$, then $g(z)=$ $z \sqrt{f\left(z^{2}\right) / z^{2}}$ belongs to $\Sigma$, and its Grunsky coefficient $\gamma_{33}$ equals $\frac{-1}{2}\left(b_{2}+\frac{1}{12} b_{0}^{3}\right)$ in terms of the coefficients of $f$. It follows that $\left|b_{2}+\frac{1}{12} b_{0}^{3}\right| \leq \frac{2}{3}$ is valid for functions in $\Sigma^{\prime}$. Next estimate

$$
\operatorname{Re}\left\{3 b_{0}+b_{2}\right\}=\operatorname{Re}\left\{b_{2}+\frac{1}{12} b_{0}^{3}\right\}+\operatorname{Re}\left\{3 b_{0}-\frac{1}{12} b_{0}^{3}\right\} \leq \frac{2}{3}+\operatorname{Re}\left\{3 b_{0}-\frac{1}{12} b_{0}^{3}\right\} .
$$

Since $\left|b_{0}\right| \leq 2$, the expression $\operatorname{Re}\left\{3 b_{0}-\frac{1}{12} b_{0}^{3}\right\}$ is bounded by

$$
\max _{|w| \leq 2} \operatorname{Re}\left\{3 w-\frac{1}{12} w^{3}\right\}=\max _{|w|=2} \operatorname{Re}\left\{3 w-\frac{1}{12} w^{3}\right\}=\max _{\theta} \operatorname{Re}\left\{6 e^{i \theta}-\frac{2}{3} e^{3 i \theta}\right\} .
$$


With $x=\cos \theta$, this becomes $\max _{-1 \leq x \leq 1}\left\{8 x-\frac{8}{3} x^{3}\right\}$, which one easily verifies is $\frac{16}{3}$. Thus we have proved the inequality $\operatorname{Re}\left\{3 b_{0}+b_{2}\right\} \leq 6$, from which it follows that $\mathscr{A}_{2}^{\prime} \leq 3$.

It is possible that the correct value for $\mathscr{A}_{2}^{\prime}$ is 1 , although the proof that is above does not give it. We shall see that a similar proof does give the value 1 if we are permitted to assume that $b_{0}$ is real.

THEOREM 6. If $f(z)=z+\sum_{n=0}^{\infty} b_{n} z^{-n}$ belongs to $\Sigma^{\prime}$ and $b_{0}$ is real, then $\operatorname{Re}\left\{s b_{0}+b_{2}\right\} \leq 2 s$ for all $s \geq 1$. Furthermore, for each $s<1$ there is a function in $\Sigma^{\prime}$ with $b_{0}$ real that violates this inequality.

PROOF. As in the previous proof, we estimate

$$
\operatorname{Re}\left\{s b_{0}+b_{2}\right\}=\operatorname{Re}\left\{b_{2}+\frac{1}{12} b_{0}^{3}\right\}+s x-\frac{1}{12} x^{3} \leq \frac{2}{3}+s x-\frac{1}{12} x^{3}
$$

where $x=b_{0}$ is real. For fixed $s \geq 1$, the maximum of $\frac{2}{3}+s x-\frac{1}{12} x^{3}$ over $-2 \leq x \leq 2$ occurs when $x=2$, and it has the value $2 s$. Thus the inequality $\operatorname{Re}\left\{s b_{0}+b_{2}\right\} \leq 2 s$ is established for all $s \geq 1$.

It does not appear to be easy to find functions in $\Sigma^{\prime}$ with $\operatorname{Re}\left\{s b_{0}+b_{2}\right\}>$ $2 s$ when $s<1$. For this purpose, we shall construct functions in $\Sigma^{\prime}$ that map onto the complement of arcs on critical trajectories of the quadratic differential $(w-1)^{2} / w d w^{2}$. That is, the omitted set will be a real interval $[0, R], 1<R<4$, plus symmetric arcs issuing from the point 1 into the upper and lower half-planes. By symmetry, such a mapping will have real coefficients. Since $(w-1)^{2} / w d w^{2} \geq 0$ on the omitted set and since $f^{\prime}$ vanishes at its tips, we may by the Schwarz reflection principle identify

$$
\frac{[f(z)-1]^{2}}{f(z)}\left[z f^{\prime}(z)\right]^{2}=\frac{(z-1)^{2}\left(z-e^{i \alpha}\right)^{2}\left(z-e^{-i \alpha}\right)^{2}}{z^{3}}
$$

for some real constant $\alpha$. Integration leads to the algebraic equation

$$
[f(z)-3] \sqrt{f(z) / z}=z-3(1+2 \cos \alpha)\left(1+z^{-1}\right)+z^{-2}
$$

for $f$. The point $R=f(1)$ determines $\cos \alpha$; that is, $(R-3) \sqrt{R}=-4-12 \cos \alpha$.

We expand the algebraic equation for $f$ near infinity to obtain

$$
\begin{gathered}
\frac{3}{2} b_{0}-3=-3(1+2 \cos \alpha), \\
\frac{3}{2} b_{1}+\frac{3}{8} b_{0}^{2}-\frac{3}{2} b_{0}=-3(1+2 \cos \alpha), \\
\frac{3}{2} b_{2}+\frac{3}{4} b_{0} b_{1}-\frac{3}{2} b_{1}-\frac{1}{16} b_{0}^{3}+\frac{3}{8} b_{0}^{2}=1 .
\end{gathered}
$$

If we consider $\cos \alpha, b_{0}, b_{1}$, and $b_{2}$ to be parametrized by $R$, then these functions are differentiable at $R=4$, and at $R=4$ we have $\cos \alpha=\frac{-1}{2}, b_{0}=2, b_{1}=1$, $b_{2}=0,(d / d R) \cos \alpha=\frac{-3}{16},(d / d R) b_{0}=\frac{3}{4},(d / d R) b_{1}=\frac{3}{4},(d / d R) b_{2}=\frac{-3}{4}$, and $(d / d R)\left[s b_{0}+b_{2}\right]=\frac{3}{4}(s-1)$. As a consequence, for each fixed $s<1$ the expression $s b_{0}+b_{2}$ decreases to $2 s$ as $R$ approaches 4 from below. Thus, for each $s<1$ there is an $R$, close to 4 , and a corresponding function in $\Sigma^{\prime}$ with real coefficients for which $s b_{0}+b_{2}>2 s$.

Just as in the last sentence of Theorem 1 , the inequality $\operatorname{Re}\left\{s b_{0}+\left|b_{n}\right|\right\} \leq 2 s$ is valid under some constraints. 
THEOREM 7. If $n \geq 2$, if $s \geq 4 / \sqrt{n}$, and if $f(z)=z+\sum_{n=0}^{\infty} b_{n} z^{-n}$ is a function in $\Sigma^{\prime}$ such that $\operatorname{Re}\left\{b_{0}\right\} \leq 2-8 /\left(n s^{2}+16\right)$, then $\operatorname{Re}\left\{s b_{0}+\left|b_{n}\right|\right\} \leq 2 s$.

ProOF. Denote $x=\operatorname{Re}\left\{b_{0}\right\}$ and $y=\operatorname{Re}\left\{b_{1}\right\}$. The area theorem, $\sum_{n=1}^{\infty} n\left|b_{n}\right|^{2} \leq$ 1 , implies that $n\left|b_{n}\right|^{2} \leq 1-y^{2}$. We use also the inequality $4 x-y \leq 7$ from part (b) of Lemma 2. If $x \geq 7 / 4$, then $y^{2} \geq(4 x-7)^{2}$, and it follows that $n\left|b_{n}\right|^{2} \leq 1-(4 x-7)^{2}=$ $8(2-x)(2 x-3)$. Now we have $\operatorname{Re}\left\{s b_{0}+\left|b_{n}\right|\right\} \leq s x+\sqrt{8(2-x)(2 x-3) / n}$, and the latter expression is at most $2 s$ whenever $\sqrt{8(2-x)(2 x-3) / n} \leq s(2-x)$. For $x \leq 2$ this will be the case whenever $8(2 x-3) / n \leq s^{2}(2-x)$ or $x \leq 2-8 /\left[n s^{2}+16\right]$. Thus the theorem is proved in case $7 / 4 \leq x \leq 2-8 /\left[n s^{2}+16\right]$. If $x<7 / 4$ and $s \geq 4 / \sqrt{n}$, then $\operatorname{Re}\left\{s b_{0}+\left|b_{n}\right|\right\} \leq 7 s / 4+1 / \sqrt{n} \leq 2 s$.

Each function in $\Sigma^{\prime}$ satisfies $\operatorname{Re}\left\{b_{0}\right\} \leq 2$, and equality occurs only for the function $k$. Since $2-8 /\left[n s^{2}+16\right]$ approaches 2 whenever either $n \rightarrow \infty$ or $s \rightarrow \infty$, this theorem has two noteworthy consequences: (i) If $s$ is positive and fixed, then the inequality $\operatorname{Re}\left\{s b_{0}+\left|b_{n}\right|\right\} \leq 2 s$ is valid for all $n$ sufficiently large. (ii) If $n \geq 2$ is fixed, then the inequality $\operatorname{Re}\left\{s b_{0}+\left|b_{n}\right|\right\} \leq 2 s$ is valid for all $s$ sufficiently large. Unfortunately, how large $n$ must be in the first case and how large $s$ must be in the second case depend on $f$.

\section{REFERENCES}

1. P. R. Garabedian and M. Schiffer, A coefficient inequality for schlicht functions, Ann. of Math. (2) 61 (1955), 116-136.

2. J. A. Jenkins, On certain coefficients of univalent functions, Analytic Functions, Princeton Univ. Press, Princeton, N. J., 1960, pp. 159-194.

3. W. E. Kirwan and G. Schober, New inequalities from old ones, Math. Z. 180 (1982), 19-40.

4. Y. J. Leung and G. Schober, Low order coefficient estimates in the class $\Sigma$, Ann. Acad. Sci. Fenn. Ser. A.I. 11 (1986), 39-62.

5. __ High order coefficient estimates in the class $\Sigma$, Proc. Amer. Math. Soc. 94 (1985), 659664.

6. $\ldots$, On the structure of support points in the class $\Sigma$, J. Analyse Math. 46 (1986), 176-193.

7. M. Ozawa, Coefficient estimates for the class $\Sigma$, Kodai Math. J. 9 (1986), 123-133.

8.

9. G. Schober, Univalent functions-selected topics, Lecture Notes in Math., vol. 478, SpringerVerlag, Berlin and New York, 1975.

10. __ Some conjectures for the class $\Sigma$, Contemp. Math. vol., 38, Amer. Math. Soc., Providence, R. I., 1985, pp. 13-21.

11. _ Kirwan's conjecture, Proceedings of the Special Year in Complex Analysis I, Lecture Notes in Math., vol. 1275, Springer-Verlag, Berlin and New York, 1987, pp. 266-271.

12. G. Schober and J. K. Williams, On coefficient estimates and conjectures for the class $\Sigma$, Math. Z. 186 (1984), 309-320.

Department of Mathematics, KuWAit UNiversity, KUWAit

Department of Mathematics, Indiana UNiVERSity, Bloomington, Indiana 47405 